\title{
Synthesis of 1,5-disubstituted tetrazoles containing propargyl moiety
}

\author{
Ángel Rentería-Gómez and Rocío Gámez-Montaño * \\ Departamento de Química, División de Ciencias Naturales y Exactas, \\ Universidad de Guanajuato, Noria Alta S/N, Col. Noria Alta, \\ Guanajuato, C.P. 36050 Guanajuato, México.e-mail: rociogm@ugto.mx
}

\begin{abstract}
:
A series of nine new 1,5-disubstituted-1 $H$-tetrazoles were synthesized via Ugi-azide in moderate to excellent yields (80-95\%) using propargyl amine as component varying the aldehyde and isocyanide components. 1,5-DS-T are useful heterocyclic moieties present in many bioactive compounds and drugs. Moreover 1,5-DS-T are used as bidentate ligands in coordination chemistry, metal-organic framework science, bioimaging, photo-imaging, explosives, propellants, and high energy materials.
\end{abstract}

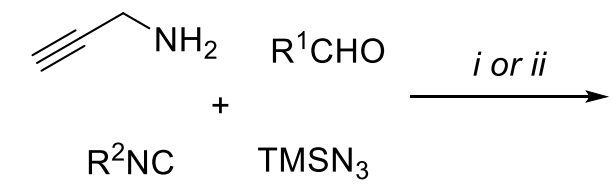

i) $\mathrm{MeOH}, 8-18 \mathrm{~h}$, r.t.

ii) $\mathrm{MeOH}, 2-4 \mathrm{~h}$, sonication

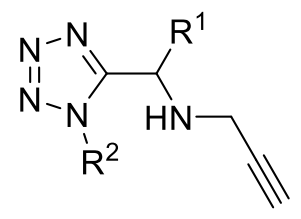

9 examples

i) $80-95 \%$

ii) $5-83 \%$

Keywords: Tetrazole, Propargyl moiety, multicomponent reaction.

\section{Introduction}

Multicomponent reactions (MCR) are useful tools toward libraries of new compounds. ${ }^{1}$ MCR allow the synthesis of target scaffolds in one pot manner. Tetrazoles are heterocyclic frameworks present in many biological compounds and drugs. As examples, cilostazol, latamoxef and BMS-317180. ${ }^{2}$ Also, tetrazole-based compounds have been used in medicinal chemistry, ${ }^{3}$ materials chemistry, ${ }^{4}$ organometallic and coordination chemistry, ${ }^{5}$ and organocatalysis. ${ }^{6,7}$ The biological activities of compounds containing tetrazole rings are usually attributed to their ability to mimic ${ }^{8} \mathrm{cis}$-amide bond of peptides. ${ }^{9}$

Various approaches for accessing to 1,5-DS-T have been described. However, the main synthetic methods are intermolecular cycloaddition [3+2] between azides and nitriles, and isocyanide-based multicomponent reactions (I-MCR). ${ }^{10}$

The Ugi-Azide reaction (UAR) was reported first by Ugi in 1961. The carboxylic acid used in the classical Ugi reaction is replaced by hydrazoic acid (generated in situ from $\mathrm{NaN}_{3} / \mathrm{TMSN}_{3}$ ). The proposed mechanism of this one-pot 4-CR involves the next stages: a condensation of amines with carbonyl compounds gives the intermediate $\mathbf{6}$, which after protonation by $\mathrm{HN}_{3}$ reacts via nucleophilic 
addition with isocyanide to afford compound $\mathbf{8}$. Then, this latter one reacts with $\mathrm{N}_{3}{ }^{-}$to give the intermediate 9. Finally, an intramolecular cyclization occurs to afford 1,5-DS-T 10 (Scheme 1). ${ }^{11}$

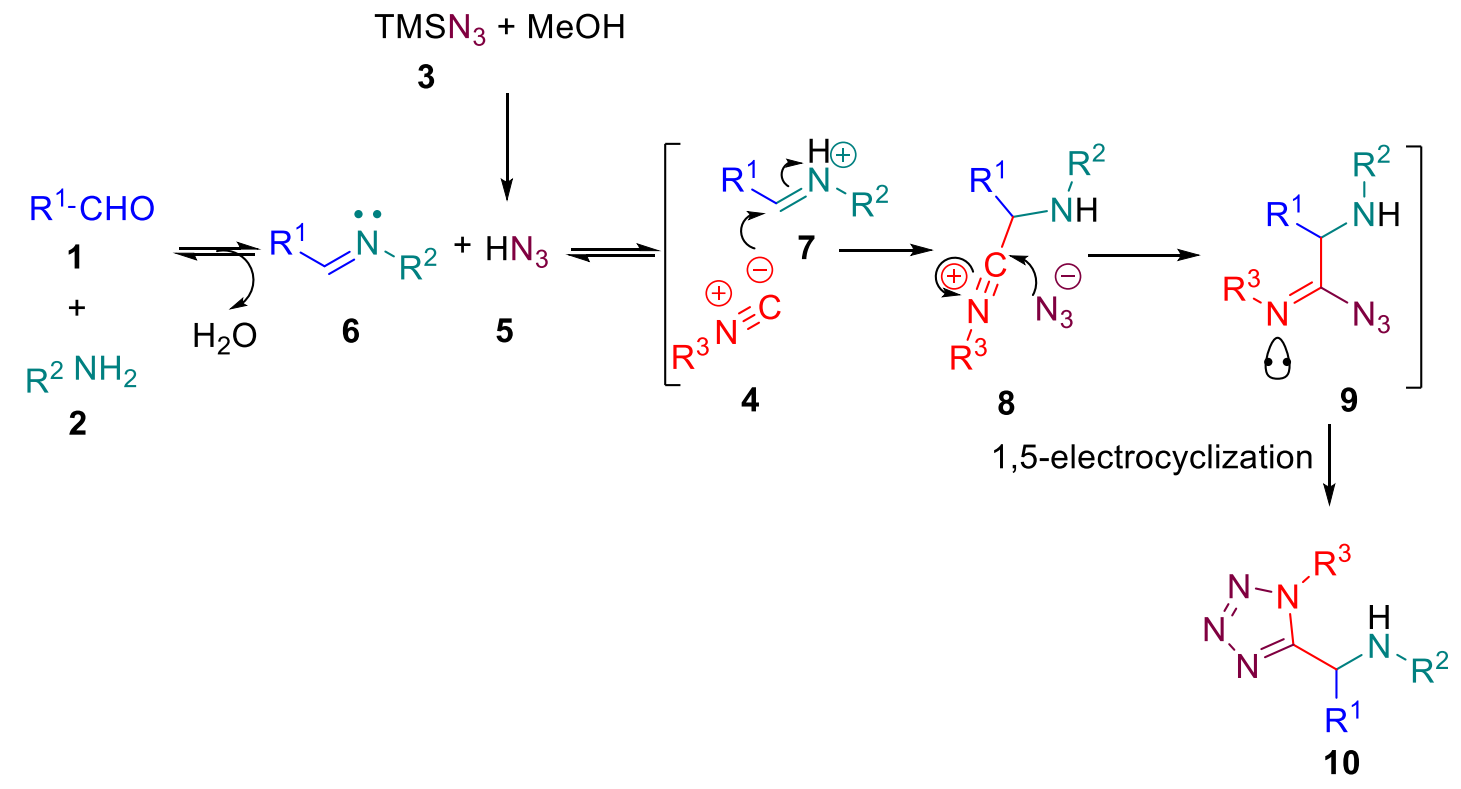

Scheme 1. Ugi-azide reaction mechanism.

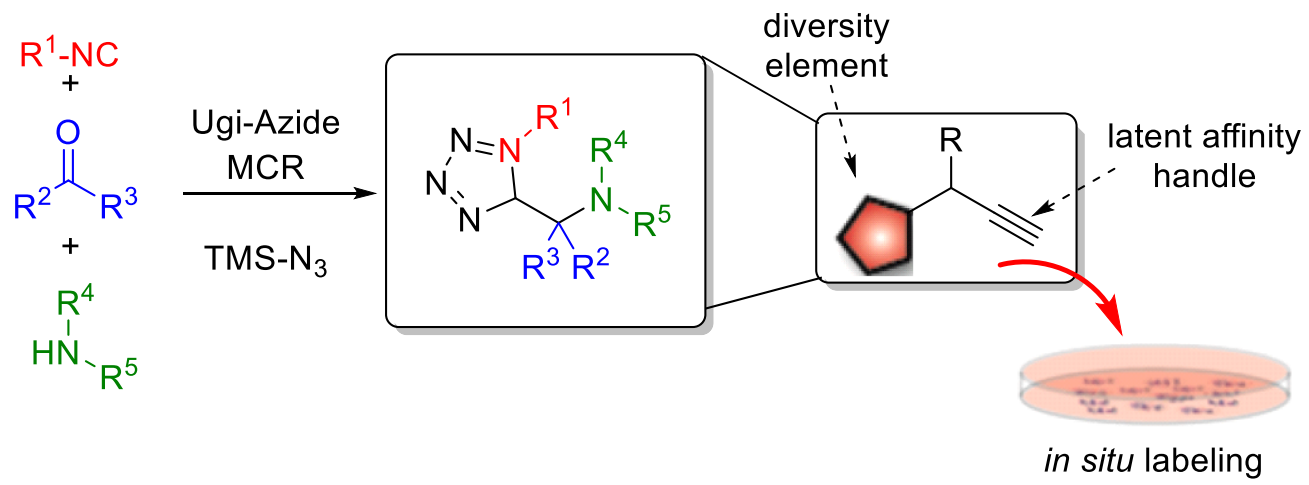

Scheme 2. Alkyne groups for protein photocrosslinking containing in tetrazole 1,5-disubstituted.

In this work, we described the synthesis of new 1,5-disubstituted- $1 H$-tetrazoles containing propargyl moiety, the literature survey revealed that this group is used to increase the covalent interaction, detection and identification of protein targets (Scheme 2). ${ }^{12}$

\section{Results and Discussion}

In this work, we describe the synthesis of nine new 1,5-disubstituted-1 $H$-tetrazoles (15a-i) containing the propargyl moiety in good to excellent yields (80-95\%) via a catalyst free Ugi-azide reaction from 
propargylamine 11, aldehydes 12, isocyanides 13 and $\mathrm{TMSN}_{3} 14$ in $\mathrm{MeOH}$ at room temperature, (Scheme 3).

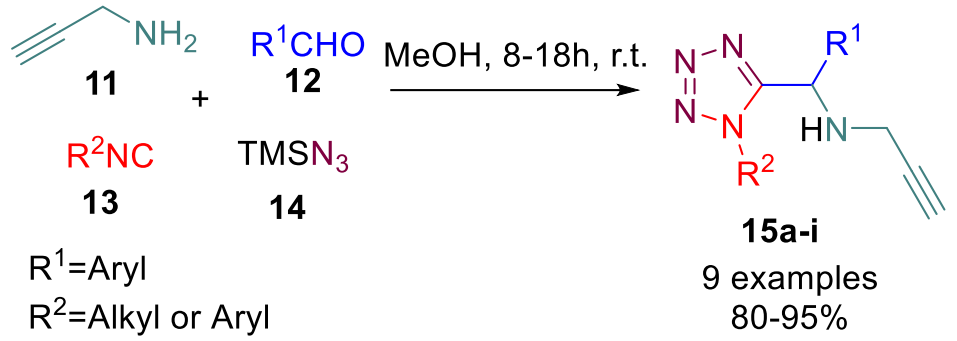

Scheme 3. Strategy of synthesis 1,5-disubstituted- $1 H$-tetrazoles

As depicted in Scheme 3, most of the compounds were obtained in good yields. For example, 15e (92\%), 15g (94\%) and 15h (95\%). Thus, yields are independent of the steroelectronic nature of substituents in the starting materials. Steric and electronic effects from isocyanide had effect in the yields of the corresponding 1,5-disubstituted- $1 H$-tetrazoles. In general, when the low nucleophilic 2,6-diMePh isocyanide was used, the yield was little lower in comparison with cyclohexil and $t$-butil isocyanide derivatives. Interestingly, when 3,4 dimethoxy aldehydes were used, good yields were found (85-95\%). As this aldehyde is deactivating for the condensation process, high nucleophilicity from propargyl amine allowed the further transformation.

Recently we have reported the first UA reaction using ultrasound irradiation (USI) to synthesize 3tetrazolyl-2-chloroquinolines in moderate to excellent yields $(33-90 \%){ }^{13}$ In this context, an experiment was performed using ultrasound (US) irradiation. The yields obtained at room temperature were higher with respect to those found using USI (5-83\%). Besides, sonication reduced the reaction time from $8-18 \mathrm{~h}$ to $2-4 \mathrm{~h}$.

\section{Conclusions}

A series of nine new 1,5-disubstituted-1 $H$ tetrazoles in good to excellent yields was synthesized, in one pot using mild conditions at room temperature. The products herein described may find application in various fields, but mainly in medicinal chemistry since they contain tetrazole moiety, which together with propargyl moiety can result in bioactive compounds. It is Noteworthy that Ugiazide reaction took place using component amine such as the propargyl amine which allowed the use of aldehydes deactivated with electrodonating in good yields. 

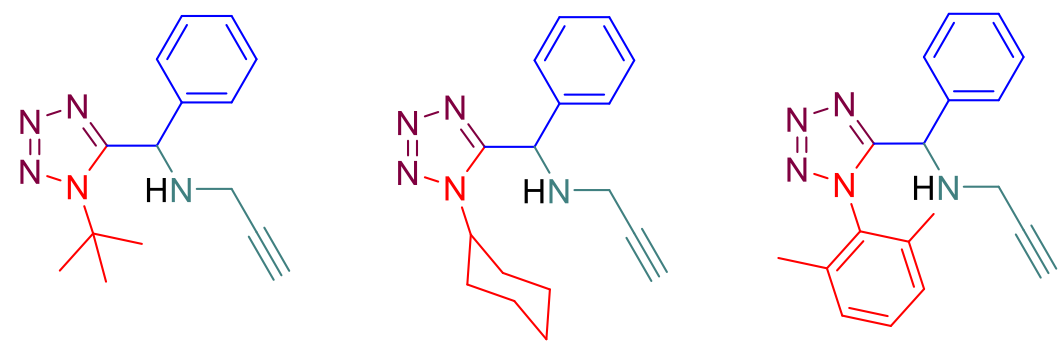

$$
\begin{gathered}
\text { r. t. }(87 \%) \\
))(75 \%) \\
15 a
\end{gathered}
$$

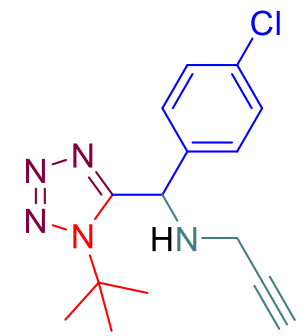

r. t. $(88 \%)$

)) $(69 \%)$

$15 b$

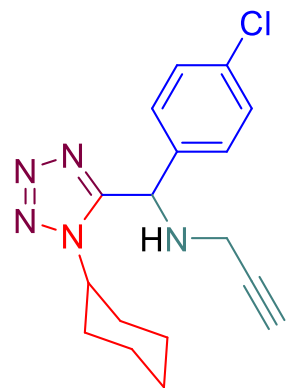

$$
\begin{aligned}
& \text { r. t. }(90 \%) \\
& ))(77 \%) \\
& 15 d
\end{aligned}
$$

$$
\begin{gathered}
\text { r. t. }(92 \%) \\
))\left(\begin{array}{c}
(75 \%) \\
15 e
\end{array}\right.
\end{gathered}
$$
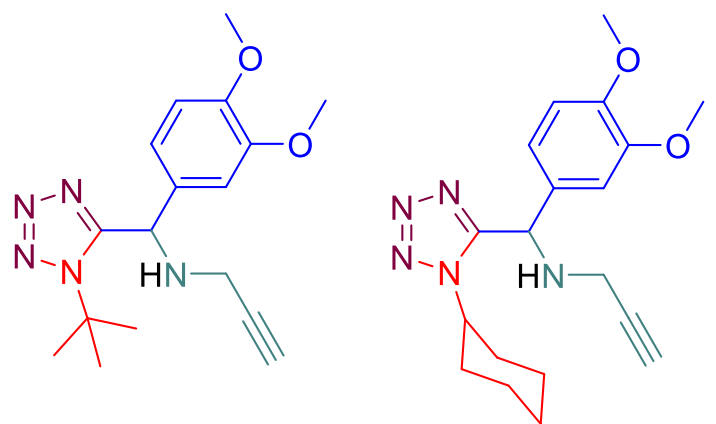

$$
\begin{aligned}
& \text { r. t. }(94 \%) \\
& \text { )) }(83 \%) \\
& 15 g
\end{aligned}
$$

r. t. $(80 \%)$

)) $(12 \%)$

$15 \mathrm{c}$

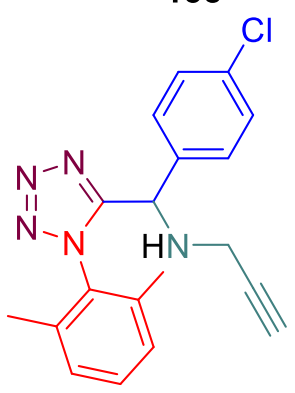

r. t. $(82 \%)$
)$))(5 \%)$
$15 f$

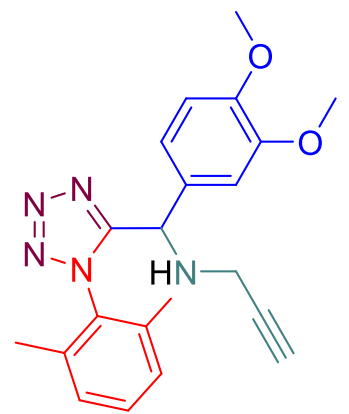

r. t. $(85 \%)$
)) $(20 \%)$
$15 i$

Scheme 4. Substrate Scope.

\section{Experimental Part}

${ }^{1} \mathrm{H}$ and ${ }^{13} \mathrm{C}$ NMR spectra were acquired on a $500 \mathrm{MHz}$ spectrometer. The solvent for NMR samples was $\mathrm{CDCl}_{3}$. Chemical shifts are reported in parts per million $(\delta / \mathrm{ppm})$. Internal reference for NMR spectra is TMS at $0.00 \mathrm{ppm}$. Coupling constants are reported in Hertz $(\mathrm{J} / \mathrm{Hz})$. Multiplicities of the signals are reported using the standard abbreviations: singlet (s), doublet (d), triplet (t), quartet (q), and multiplet (m). HRMS spectra were acquired via electrospray ionization ESI $(+)$ and recorded via the TOF method. The reaction progress was monitored by TLC and the spots were visualized under 
UV light (254 or $365 \mathrm{~nm}$ ). Flash column chromatography was performed using silica gel (230-400 mesh) and mixtures in different proportions of hexanes with ethyl acetate as mobile phase.

General method: Propargylamine 11 (1.0 M) (1.0 equiv.) aldehyde 12 (1.0 equiv.), isocyanide 13 (1.0 equiv.) and azidotrimethylsilane 14 (1.0 equiv.) were disolved in $\mathrm{MeOH}$ in a round-bottom flask equipped with a magnetic stirrer bar. The resulting mixture was stirred for $8-18 \mathrm{~h}$ under an inert nitrogen atmosphere at room temperature. The solvent was evaporated under reduced pressure until dryness, the organic layer was purified by column chromatography on silica gel using a mixture of Hex-AcOEt (4:1 V/V) as eluent to afford compounds (15a-i).

N-((1-(tert-butyl)-1H-tetrazol-5-yl)(phenyl)methyl)prop-2-yn-1-amine (15a) Yellow liquid; Yield: 87\%; $\mathrm{R}_{f}=0.12(\mathrm{Hex}-\mathrm{AcOEt}=3: 2 ; \mathrm{v} / \mathrm{v})$. Spectral data: ${ }^{1} \mathrm{H} \mathrm{NMR}\left(500 \mathrm{MHz} ; \mathrm{CDCl}_{3} ; 25^{\circ} \mathrm{C}\right.$, TMS): $\delta 7.31-7.22(\mathrm{~m}, 5 \mathrm{H}), 5.6(\mathrm{~s}, 1 \mathrm{H}), 5.55(\mathrm{~s}, 1 \mathrm{H}), 3.36(\mathrm{~s}, 2 \mathrm{H}), 2.25(\mathrm{~s}, 1 \mathrm{H}), 1.58(\mathrm{~s}, 9 \mathrm{H}) \mathrm{ppm} ;{ }^{13} \mathrm{C}$ $\mathrm{NMR}\left(126 \mathrm{MHz}, \mathrm{CDCl}_{3}, 25^{\circ} \mathrm{C}, \mathrm{TMS}\right): \delta 154.63,137.75,129.05,128.64,128.34,80.86,72.79,61.58$, 56.38, 36.02, $29.87 \mathrm{ppm}$. FT-IR (ATR) $v_{\max } / \mathrm{cm}^{-1} 3344(\mathrm{~N}-\mathrm{H}), 1237(\mathrm{~N}-\mathrm{N}=\mathrm{N})$. HRMS $[\mathrm{M}+\mathrm{H}]^{+}: \mathrm{m} / \mathrm{z}$ calcd for $\mathrm{C}_{15} \mathrm{H}_{19} \mathrm{~N}_{5}: 270.1713$; found: .270.1719

\section{Acknowledgements}

R.G.-M. is grateful for financial support from CIO-UG (009/2015), DAIP (859/2016) and CONACYT (CB-2011-166747-Q) projects. A.R.-G. (554166/290817) acknowledge CONACYTMéxico for their scholarships. All authors kindly acknowledge to National Laboratory for the instrumentation time provided (UG-UAA-CONACYT: 123732).

\section{References}

1 (a) Ugi, I.; Heck, S. Comb. Chem. High Throughput Screen. 2001, 4, 1. (b) Rentería-Gómez, A.; Islas-Jácome, A.; Díaz-Cervantes, E.; Villaseñor-Granados, T.; Robles, J.; Gámez-Montaño, R. Bioorg. Med. Chem. Lett. 2016, 26, 2333. (c) Gordillo-Cruz, R. E.; Rentería-Gómez, A.; IslasJácome, A.; Cortes-García, C. J.; Díaz-Cervantes, E.; Robles, J.; Gámez-Montaño, R. Org. Biomol. Chem. 2013, 11, 6470. (d) Cárdenas-Galindo, L. E.; Islas-Jácome, A.; Alvarez-Rodríguez, N. V.; El Kaim, L.; Gámez-Montaño, R. Synthesis 2014, 46, 49. (e) Cano, P. A.; Islas-Jácome, A.; GonzálezMarrero, J.; Yépez-Mulia, L.; Calzada, F.; Gámez-Montaño, R. Bioorg. Med. Chem. 2014, 22, 1370. (f) Cárdenas-Galindo, L. E.; Islas-Jácome, A.; Colmenero-Martínez, K. M.; Martínez-Richa, A.; Gámez-Montaño, R. Molecules 2015, 20, 1519. (g) Cortes-García, C. J.; Islas-Jácome, A.; RenteríaGómez, A. Monatsh. Chem. 2016, 147, 1277. (h) Kishore, K. G.; Basavanag, U. M. V.; Islas-Jácome, A.; Gámez-Montaño, R. Tetrahedron Lett. 2015, 56, 155. (i) Islas-Jácome, A.; González-Zamora, E.; Gámez-Montaño, R. Tetrahedron Lett. 2011, 52, 5245. (j) Islas-Jácome, A.; Cárdenas-Galindo, L. E.; Jerezano, A. V.; Tamariz, J.; González-Zamora, E.; Gámez-Montaño, R. Synlett 2012, 23, 2951. (k) Cárdenas-Galindo, L. E.; Islas-Jácome, A.; Cortes-García, C. J.; El Kaim, L.; Gámez-Montaño, R. J. Mex. Chem. Soc. 2013, 57, 283.

2 (a) Himo, F.; Demko, Z. P.; Noodleman, L. J. Org. Chem. 2003, 68, 9076 (b) Upadhayaya, R. S.; Sinha, N.; Jain, S.; Kishore, N.; Chandra, R.; Arora, S. K. Bioorg. Med. Chem. 2004, 12, 2225. (c) Gross, E. G.; Featherstone, R. M. J. Pharmacol. Exp. Ther. 1946, 87, 299 (c) Dömling, A.; Chandgude, A. L. Green Chemistry 2016, 18, 3718.

${ }^{3}$ a) V. Bavetsias, J. H. Marriott, C. Melin, R. Kimbell, Z. S. Matusiak, F. T. Boyle, A. L. Jackman, J. Med. Chem. 2000, 43, 1910; b) R. S. Upadhayaya, S. Jain, N. Sinha, N. Kishore, R. Chandra, S. K. 
Arora, Eur. J. Med. Chem. 2004, 39, 579; c) V. A. Ostrovskii, R. E. Trifonov, E. A. Popova, Russ. Chem. Bull. 2012, 61, 768.

${ }^{4}$ H. Gao, J. M. Shreeve, Chem. Rev. 2011, 111, 7377.

5 a) E. A. Popova, R. E. Trifonov, V. A. Ostrovskii, ARCIVOC 2012, 45; b) G. Aromí, L. A. Barrios, O. Roubeau, P. Gamez, Coord. Chem. Rev. 2011, 255, 485.

${ }^{6}$ a) D. A. Longbottom, V. Franckevicius, S. V. Ley, Chimia 2007, 61, 247; b) M. Bhanushali, C.-G. Zhao, Synthesis 2011, 12, 1815; c) A. G. Doyle, E. N. Jacobsen, Chem. Rev. 2007, 107, 5713; d) C. Nájera, J. M. Sansano, Chem. Rev. 2007, 107, 4584; e) M. Limbach, Chem. Biodiversity 2006, 3, 119; f) Y.-Y. Wu, Z. Chai, X.-Y. Liu, G. Zhao, S.-W. Wang, Eur. J. Org. Chem. 2009, 904; g) H. Torii, M. Nakadai, K. Ishihara, S. Saito, H. Yamamoto, Angew. Chem. 2004, 116, 2017; Angew. Chem. Int. Ed. 2004, 43, 1983; h) A. Hartikka, P. I. Arvidsson, Eur. J. Org. Chem. 2005, 4287; i) A. J. A. Cobb, D. M. Shaw, S. V. Ley, Synlett 2004, 558; j) A. J. A. Cobb, D. M. Shaw, D. A. Longbottom, J. B. Gold, S. V. Ley, Org. Biomol. Chem. 2005, 3, 84; k) N. S. Chowdari, M. Ahmad, K. Albertshofer, F. Tanaka, C. F. Barbas III, Org. Lett. 2006, 8, 2839; 1) K. R. Knudsen, C. E. T. Mitchell, S. V. Ley, Chem. Commun. 2006, 66; m) C. E. T. Mitchell, S. E. Brenner, S. V. Ley, Chem. Commun. 2005, 5346; n) A. Prieto, N. Halland, K. Anker, Org. Lett. 2005, 7, 3897; o) A. J. A. Cobb, D. A. Longbottom, D. M. Shaw, S. V. Ley, Chem. Commun. 2004, 1808; p) Y. Yamamoto, N. Momiyama, H. Yamamoto, J. Am. Chem. Soc. 2004, 126, 5962; q) D. B. Ramachary, C. F. Barbas III, Org. Lett. 2005, 7, 1577; r) S.-T. (A.) Tong, P. W. R. Harris, D. Barker, M. A. Brimble, Eur. J. Org. Chem. 2008, 164.

${ }^{7}$ (a) Katritzky, A. R.; Cai, C.; Meher, N. K. Synthesis 2007, 1204; (b) Romagnoli, R.; Baraldi, P. G.; Salvador, M. K.; Preti, D.; Aghazadeh Tabrizi, M.; Brancale, A.; Fu, X.-H.; Li, J.; Zhang, S.-Z.; Hamel, E. J. Med. Chem. 2011, 55, 475.

${ }^{8}$ a) R. J. Herr, Bioorg. Med. Chem. 2002, 10, 3379; b) J. Roh, K. Vávrová, A. Hrabálek, Eur. J. Org. Chem. 2012, 6101.

${ }^{9}$ a) L. V. Myznikov, A. Hrabalek, G. I. Koldobskii, Chem. Heterocycl. Compd. 2007, 3; b) B. C. H. May, A. D. Abell, J. Chem. Soc. Perkin Trans. 1 2002, 172; c) A. Rajasekaran, P. P. Thampi, Eur. J. Med. Chem. 2004, 39, 273.

${ }^{10}$ Katritzky, A. R.; Cai, C.; Meher, N. K. Synthesis 2007, 1204.

${ }^{11}$ Ugi, I.; Steinbruckner, C. Chem. Ber. 1961, 94, 734.

${ }^{12}$ Kambe, T.; Correia, B. E.; Niphakis, M. J.; Cravatt, B. F. J. Am. Chem. Soc. 2014, 136, 10777.

${ }^{13}$ Unnamatla-Basavanag, M. V.; Islas-Jácome, A.; Quezada-Soto, A.; Ramírez-López, S. C.; FloresAlamo, M.; Gámez-Montaño, R. J. Org. Chem. 2016, 81, DOI: 10.1221/acs.joc.6b01576. 